\title{
Phenotypic characterization of skeletal abnormalities of Osteopotentia mutant mice by micro-CT: a descriptive approach with emphasis on reconstruction techniques
}

\author{
Frank W. Roemer • Andreas Mohr • Ali Guermazi • \\ Yebin Jiang • Philipp Schlechtweg • Harry K. Genant • \\ Michael L. Sohaskey
}

Received: 17 September 2010 /Revised: 2 December 2010 / Accepted: 7 December 2010 /Published online: 5 January 2011

(C) The Author(s) 2011. This article is published with open access at Springerlink.com

\begin{abstract}
Purpose The novel protein osteopotentia (Opt) has recently been described as an essential regulator of postnatal osteoblast maturation and might possibly be responsible for some of the rarer types of osteogenesis imperfecta. Our aim was the evaluation of micro $\mathrm{CT}$ for the qualitative morphological assessment of skeletal abnormalities of Osteopotentia-mutant mice in comparison to radiography and histology.

Materials and methods Four homozygous mice with insertional mutations in the $O p t$ gene and three wild-type controls were examined ex vivo using radiography and micro-CT. Two of the homozygous animals were evaluated histologically (trichrome reagent). For the micro-CT evaluation three-dimensional (3D) surface reconstructions and
\end{abstract}

\section{F. W. Roemer}

Department of Radiology, Klinikum Augsburg,

86156 Augsburg, Germany

F. W. Roemer $\cdot$ A. Guermazi

Quantitative Imaging Center,

Boston University School of Medicine,

Boston, MA 02118, USA

F. W. Roemer $\cdot$ A. Mohr · A. Guermazi $\cdot$ Y. Jiang $\cdot$ H. K. Genant Osteoporosis and Arthritis Research Group,

University of California, San Francisco,

San Francisco, CA 94117, USA

\section{A. Mohr}

Department of Radiology, Sligo General Hospital,

Sligo, Ireland

\section{Y. Jiang}

Osteoporosis and Arthritis Laboratory, Musculoskeletal Division,

Department of Radiology,

University of Michigan Medical School,

Ann Arbor, MI 48109, USA two-dimensional (2D) multiplanar reformations (MPRs) were applied.

Results The Opt-homozygous mice exhibited severe growth. The radiographic examinations showed osteopenia and fractures with hypertrophic callus formation and pseudarthroses of the forelimbs and ribs. Micro-CT confirmed these findings and was able to demonstrate additional fractures especially at smaller bones such as the metacarpals and phalanges. Additional characterization and superior delineation of cortices and fracture fragments was achieved by $2 \mathrm{D}$ MPRs. Histological correlation verified several of these imaging findings.

Conclusion Micro-CT is able to screen Opt-mutant mice for osseous pathologies and furthermore characterize these anomalies. The modality seems superior to conventional

P. Schlechtweg

Department of Radiology, University of Erlangen,

Erlangen, Germany

H. K. Genant

CCBR-SYNARC, Inc.,

San Francisco, CA 94105, USA

M. L. Sohaskey

Department of Molecular and Cell Biology and Center for Integrative Genomics, University of California, Berkeley, Berkeley, CA 94720, USA

F. W. Roemer $(\bowtie)$

Department of Radiology, Boston University Medical Center,

FGH Building, 3rd floor 820 Harrison Avenue,

Boston, MA 02118, USA

e-mail: froemer@bu.edu 
radiography, but is not able to demonstrate cellular pathology. However, histology is destructive and more time- and material-consuming than micro-CT. Additional information may be gathered by 2D MPRs.

Keywords Animal model $\cdot$ Micro CT $\cdot$ Bone .

Radiography $\cdot$ Fracture $\cdot$ Osteogenesis imperfecta

\section{Introduction}

Osteogenesis imperfecta (OI) is a genetic disorder of the bone extracellular matrix. The hallmark features of OI are bone fragility and susceptibility to fractures. About $90 \%$ of OI cases are caused by dominant mutations in one of the two genes encoding type I procollagen, COL1A1 and COL1A2. The novel protein osteopotentia (Opt) has recently been described as an essential regulator of postnatal osteoblast maturation and might possibly be responsible for some of the rarer types of OI [1,2]. Loss of Opt leads to impaired type I collagen synthesis and a consequent failure of bone modeling [2], suggesting that Opt deficiency may be responsible for some rarer forms of recessive OI $[1,2]$.

Mice homozygous for an insertional mutation in the $O p t$ gene die predominantly at birth of undetermined causes. However, a subset survives long enough to develop striking skeletal abnormalities similar to those seen in humans with more severe forms of OI [2].

We employed Opt-mutant mice to demonstrate the ability of micro-CT $(\mu \mathrm{CT})$ to characterize the phenotypic skeletal pathology in comparison to conventional radiography and traditional histology. The power of $\mu \mathrm{CT}$ scanning to obtain such information is illustrated and the importance of two-dimensional (2D) multiplanar reconstructions (MPRs) in addition to more commonly employed three-dimensional (3D) surface-rendered images is emphasized.

\section{Materials and methods}

The study was approved by the institutional review boards of the University of California at San Francisco and Berkeley.

Animals

Four Opt-homozygous mutant mice and three wild-type controls, all 20-day-old females, were selected for these studies. Animals were maintained in a facility in accordance with the institutional guidelines on animal research and were euthanized by means of $\mathrm{CO}_{2}$ inhalation.
Conventional radiography

Radiography in two planes was performed on all animals. All radiographs were acquired at $70 \mathrm{kVp}$ with Kodak Diagnostic Film, X-OMAT (Industrial Film), with $5.94 \mathrm{~s}$ exposure time, a spatial resolution of $10 \mathrm{lp} / \mathrm{mm}$ and at $68 \mathrm{~cm}$ focus-to-film distance. Assessment of radiographic images was performed with the help of a magnifying glass, and several skeletal abnormalities focusing on fractures, sclerosis and deformities were recorded.

\section{Micro-CT}

The $\mu \mathrm{CT}$ system is a cone beam tomograph $(\mu \mathrm{CT} 40$; Scanco Medical, Bassersdorf, Switzerland) with a microfocus X-ray tube providing a $10-\mu \mathrm{m}$ focal spot. The source produces a cone beam that is detected by a charge coupled device array. The specimens were scanned in the transverse plane, mounted in a cylindrical sample holder with a current of $144 \mu \mathrm{A}$, a voltage of $55 \mathrm{kVp}$, at an isotropic resolution of $30 \mu \mathrm{m}$ and a field of view of $30.7 \mathrm{~mm}$ (image matrix $1,024 \times 1,024$ pixels). Scanning times varied between 4.2 and $7.8 \mathrm{~h}$ depending on the coverage of anatomy and animal size respectively. Image reconstruction times were 8.4 to $15.6 \mathrm{~h}$. 3D surface reconstructions were performed using the $\mu \mathrm{CT}$ software at the same threshold.

Additional MPRs were performed on a desktop computer using customized software for image analysis [3, 4]. The image datasets were exported in DICOM 3.0 format from the micro-CT scanner. Slice thickness of these reconstructions was $30 \mu \mathrm{m}$. The images were evaluated for appearance and detectability of bone pathology.

\section{Histology}

Two of the mutant littermates were sectioned and sections were stained by trichrome reagent (collagen in cartilage stains light blue, collagen in bone stains dark blue, muscle fibers and cellular cytoplasm stain red). Multiplanar reconstructions of $\mu \mathrm{CT}$ data were employed to compare directly with the histological sections.

\section{Results}

All Opt-mutant mice exhibited severe growth retardation. In no instance did any of the control mice exhibit any of the skeletal abnormalities evident in the Opt mutants.

Conventional radiography

Detection of skeletal pathology was impaired on conventional radiographs due to limitations in resolution. Optical 
magnification improved detectability slightly, but diagnosis of fractures was only possible for the femur, forearm and some ribs as evidenced by callus formation (Fig. 1). All Opt-mutant mice exhibited fractures of the distal forelimbs and in three of these mice fractures of the proximal humerus were suspected on the conventional radiographs.

\section{Micro-CT}

Fractures of the radius and ulna as well as of the proximal humerus were observed on $\mu \mathrm{CT}$. All of these fractures showed marked callus formation and were superiorly visualized by the $2 \mathrm{D}$ MPRs in comparison to the $3 \mathrm{D}$ surface reconstruction (Fig. 2). Dependent on the threshold applied the 3D surface reconstructions showed some of the pathologies, while the multiplanar reconstructions superiorly depicted the fracture characteristics. Due to the immaturity of the skeleton, epiphyseal ossification centers could be easily distinguished for the metacarpals and phalanges, which were only depicted on the MPRs. Marked sclerosis of the metacarpal epiphyses in comparison to the controls was evident (Fig. 2a).

\section{Traditional histology}

The histological sections verified several of the diagnosed skeletal abnormalities. Examples are shown in Fig. 3. The histologically depicted fractures were all diagnosed by $\mu \mathrm{CT}$, although the destructive approach of histology after decalcification led to partially altered bone contours in comparison to the $\mu \mathrm{CT}$ images (Fig. 3a). Correlation of a metacarpal II fracture with $\mu \mathrm{CT}$ results is presented in
Fig. 3b. While the 3D surface display $\mu \mathrm{CT}$ reconstruction barely depicts the fracture line, the coronal MPR clearly demonstrates the course of the fracture line.

\section{Discussion}

Micro-CT has become a powerful technique in nondestructive 3D imaging and morphometric analysis. This method allows for extremely high-resolution imaging and quantitative analysis due to high matrix and isotropic voxel size that can be as low as 5 microns. The major applications of micro-CT to date have been the analysis of bone [5-8], vascular and parenchymal microstructure in animal models $[9,10]$, and the characterization of the phenotype of transgenic and knockout animal models during pre-clinical investigations [11, 12]. Further applications include dental research, including measurement of enamel thickness, root canal morphology, evaluation of root canal preparation, craniofacial skeletal structure, and other parameters [13].

The ability to demonstrate skeletal pathology in three dimensions offers a means of assessing phenotypic alterations in mutant mice. The technique allows for a level of assessment that lies between gross inspection and radiography on one side and histological analysis on the other. Histology demonstrates microscopic changes, including cellular alterations, but is destructive and restricted to 2Dsampling of $3 \mathrm{D}$ structures. $\mu \mathrm{CT}$ is able to visualize anatomical structures without the physical disruption of the sample. Post-processing procedures allow for 3D reconstructions as well as 2D MPRs or other reconstructions in any given plane without loss of information. An

Fig. 1 Whole-body radiographs of 20-day-old control (left) and Opt-deficient (right) animals. No skeletal pathology is apparent in the control; by contrast, fractures of the forelimbs are apparent in the mutant (white arrows). Hyperplastic callus formation is also shown at the paravertebral portion of the ribs (black arrows). These images contain insufficient spatial resolution for detailed analysis of bony pathologies

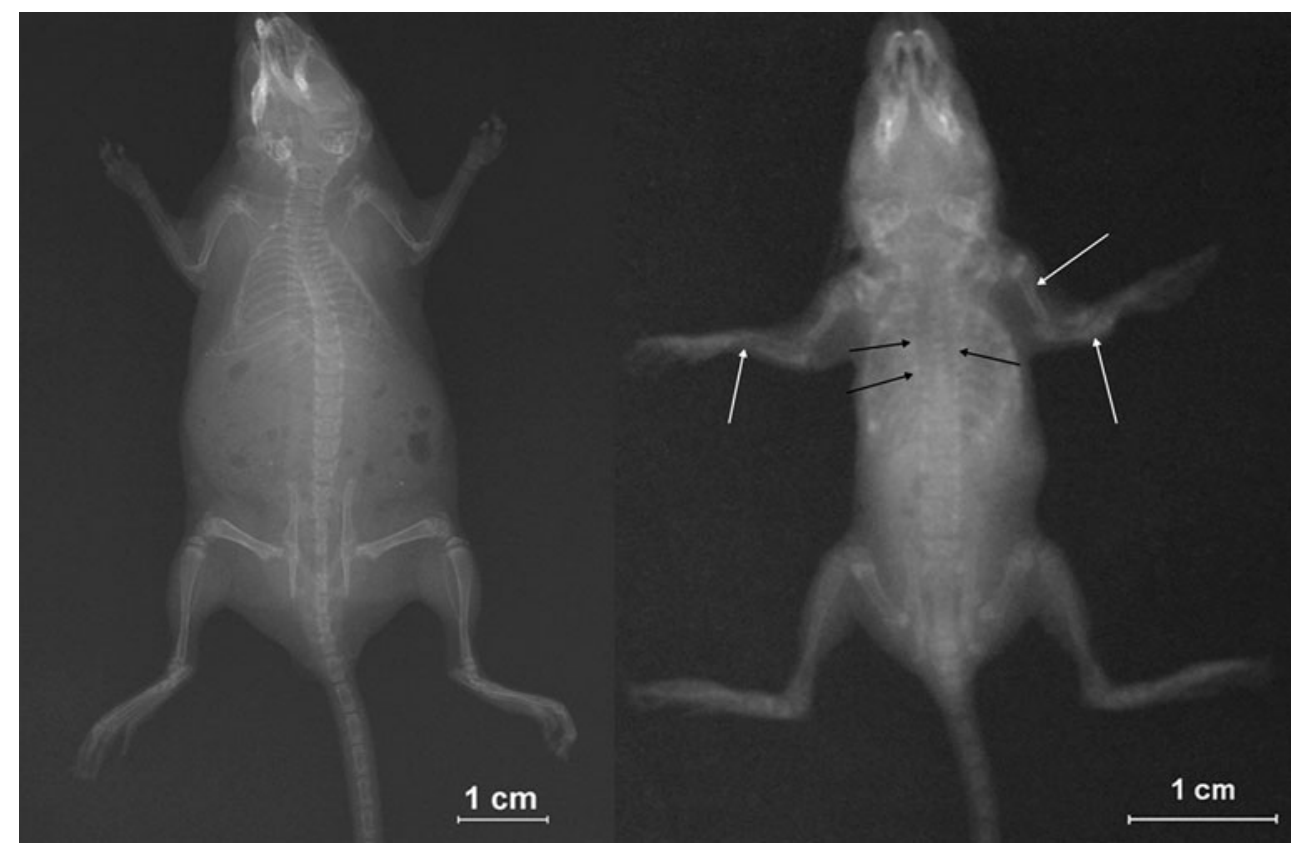




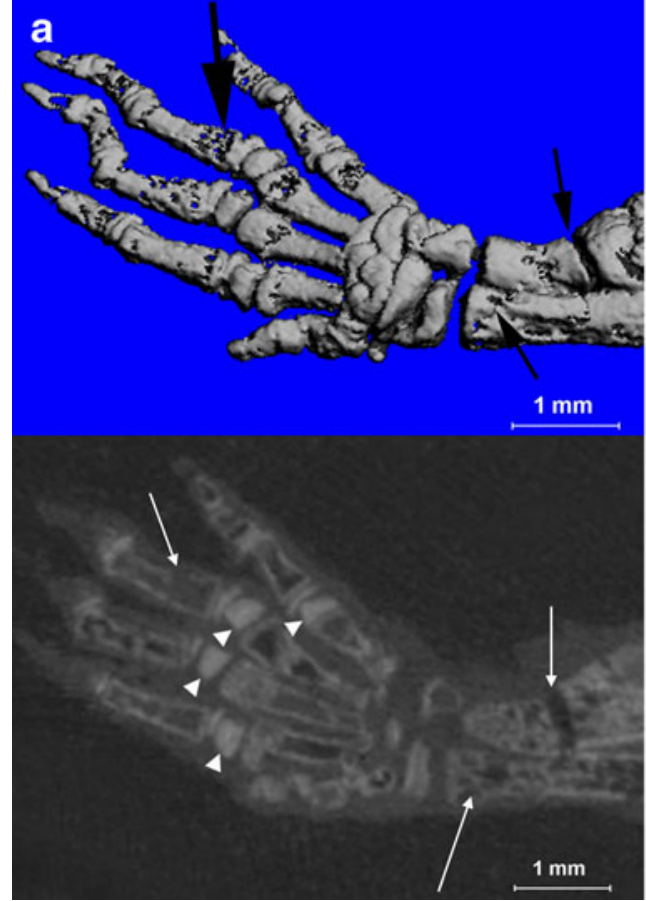

Fig. 2 Micro-CT of forelimbs from 20-day-old Opt-deficient animals. a Ulnar and radial fractures are depicted in the 3D surface reconstruction (upper image) as well as in the 2D coronal multiplanar reformation (MPR; lower image). Fractures of the distal ulna and radius are seen in the 3D surface reconstruction (small black arrows). In addition, a fracture at the base phalanx of DII is suspected (large black arrow). Cortical disruption is superiorly visualized in the MPR (white arrows). Sclerotic epiphyses are seen at the metacarpals, which

excellent example of a qualitative characterization of a mutant mouse model was presented by Ford-Hutchinson and co-workers [14]. However, as in other comparable studies, only threshold-dependent 3D surface reconstructions were employed to visualize skeletal pathology and no histological correlation was provided $[11,15]$. While the $3 \mathrm{D}$ surface-rendered reconstructions give a good overview of deformities and bone shape, in general intraosseous and cortical details are not visualized. In our study customized reconstruction software was employed to generate 2D MPRs of the original dataset on a desktop computer system $[3,4]$. This allowed for superior characterization of the observed skeletal abnormalities. To our knowledge this has not been performed in $\mu \mathrm{CT}$ analysis before, but multiple publications have proved its value for the analysis of skeletal structures in clinical CT settings, especially since the introduction of multislice CT systems $[16,17]$.

Advantages of $\mu \mathrm{CT}$ compared with $\mu \mathrm{MRI}$ or $\mu$ PET are its widespread availability in research institutions, its superior cost-efficiency, its well-known capacities for analysis and visualization of bone structure, and the possibility of multiplanar reconstructions with the same resolution as the original axial data set. Limitations of the technique include inferior

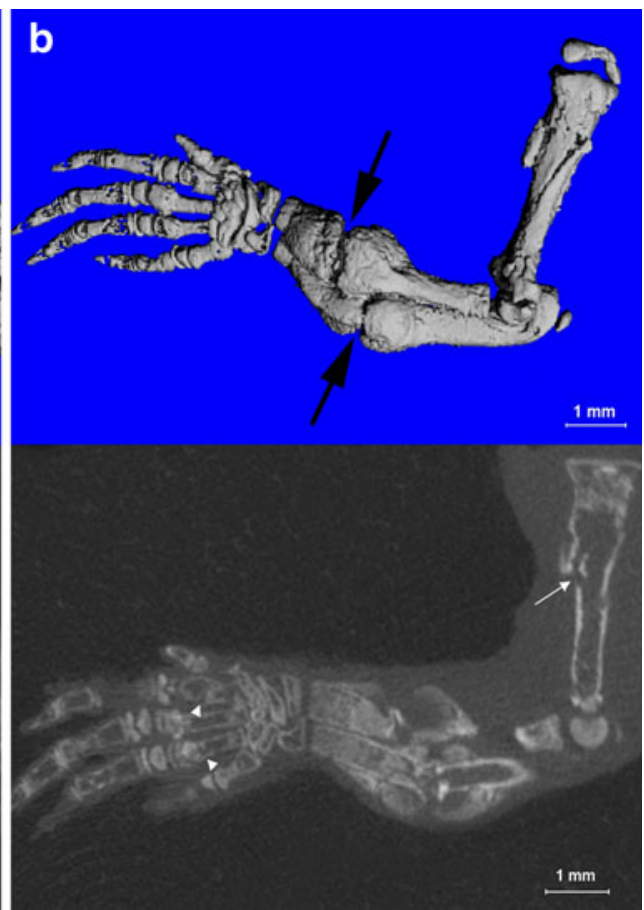

were only visualized on the MPR (arrowheads). b Fractures with cortical disruption are clearly shown for the distal radius and ulna in the $3 \mathrm{D}$ reconstruction (upper image). Additional fractures of the proximal humerus and possibly some metacarpal bones were suspected. Additional information is provided by the 2D MPR (lower image), which verified these suspected findings. A definite cortical discontinuity is shown at the humerus (arrow) and the metacarpals (arrowheads)

soft-tissue contrast, its inability to visualize cellular detail, and relatively long scanning periods [18]. Excellent overviews of the technical background and applications of $\mu \mathrm{CT}$ were presented recently $[19,20]$.

The ability to non-invasively follow changes over time in vivo will further establish $\mu \mathrm{CT}$ as a standard evaluation technique for such studies [21]. While the benefits of in vivo $\mu \mathrm{CT}$ imaging are clear, additional issues must be considered that do not have an impact on ex vivo scanning. First, the potential effects of ionizing radiation on the body region of interest (e.g., bone development and growth) must be assessed. A second potential complication of in vivo imaging is that respiratory movement while the animal is scanned under anesthesia may decrease image quality. Finally, the ability to precisely track alterations in local bone architecture and growth requires the ability to accurately register images captured over time [22].

As X-ray traverses the body, a certain amount of the radiation is absorbed, which in biological samples may cause some damage. This is generally no problem for ex vivo measurements of samples, but must be taken into account when imaging live animals. The exact dose deposition depends on many factors, such as energy, total 


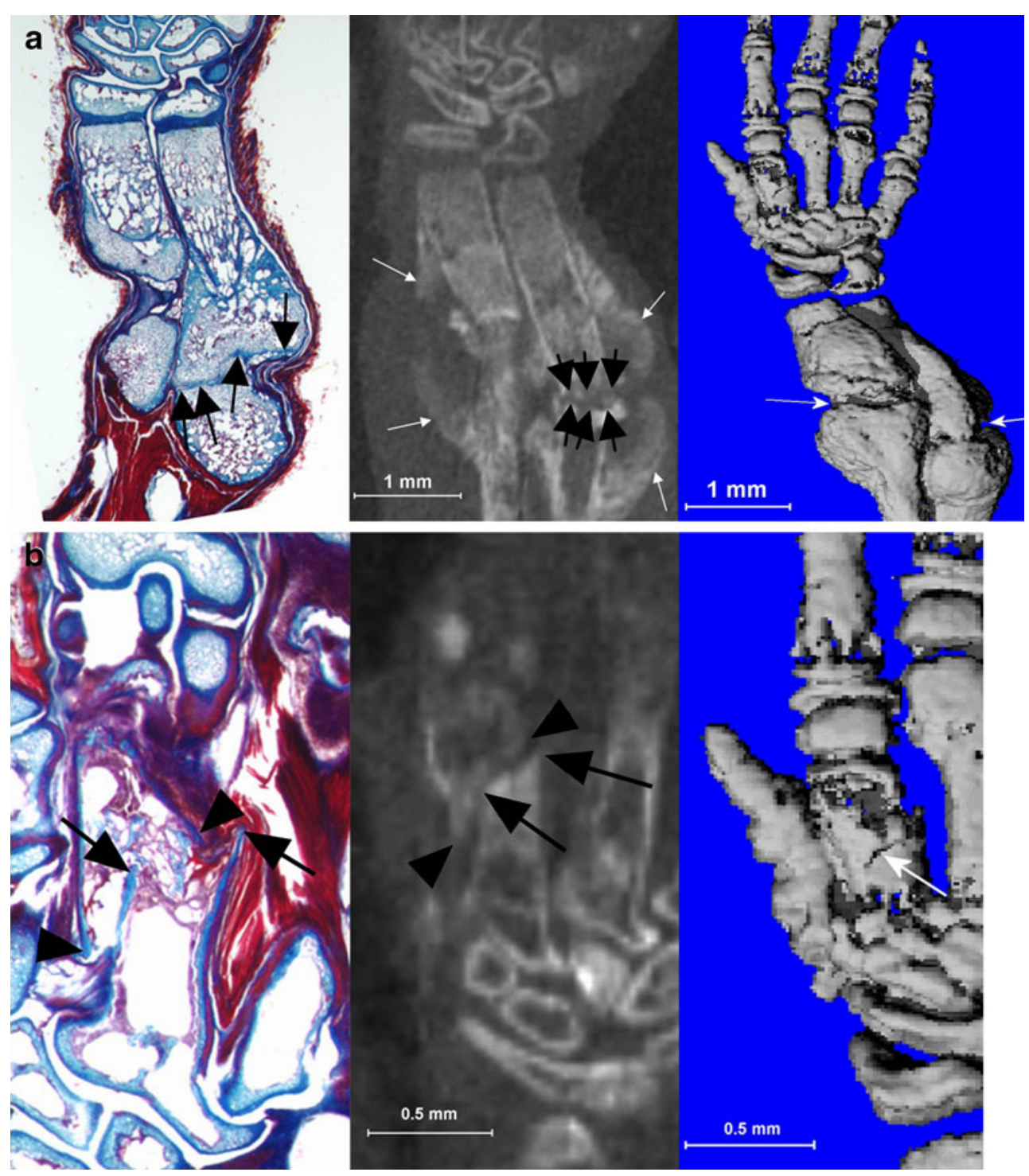

Fig. 3 A 20-day-old Opt-deficient animal. Histological correlation. a Histological coronal section (left image), 10- $\mu \mathrm{m}$ thickness, trichrome stain (collagen in cartilage is depicted with light blue, collagen in bone stains dark blue, muscle fibers and cellular cytoplasm stain red), depicts a fracture in the process of healing visualized as a dark blue band (black arrows). Corresponding coronal 2D $\mu \mathrm{CT}$ MPR (middle image) clearly shows the ulnar fracture (black arrows). In addition, hyperplastic callus formation is observed (white arrows). Note that an exact corresponding reconstruction of the $\mu \mathrm{CT}$ data to the histological image is not possible as the sectioning procedure performed after $\mu \mathrm{CT}$ scanning slightly altered the structure of the forelimb. The 3D surface reconstruction (right image) only yields inferior information content in comparison to coronal MPR. White arrows point toward the fracture lines. b Histological coronal section, $10-\mu \mathrm{m}$ thickness, trichrome stain (left image), shows an oblique fracture of the diaphyseal metacarpal II of the right forelimb without callus formation. The arrowheads point to the distal cortices of the fractured metacarpal, the arrows point toward the proximal cortices of the fracture. The 2D coronal reformation of the $\mu \mathrm{CT}$ data (middle image) shows the fracture line in similar fashion (arrowheads depict the distal part of the fracture, arrows depict the proximal part of the fracture). The 3D surface reconstruction (right image) only inferiorly delineates the oblique metacarpal fracture (white arrow points toward the fracture line)

For this reason it is of great importance to properly fix the sample in the sample holder so that no movement during the measurement is possible.

The mouse model we employed for illustration of the ability of $\mu \mathrm{CT}$ to characterize skeletal pathology is the result of an insertional mutation in the $O p t$ gene. Surviving homozygous mutant animals exhibit phenotypic features resembling severe
Imaging times in our study were relatively long due to the high resolution required for detailed skeletal analysis. 
OI. Concerning bone structural parameters $\mu \mathrm{CT}$ revealed significantly reduced trabecular bone volume and dramatically thinner cortices in long bones, despite only mild perturbations to the organization of growth plate chondrocytes. Trabecular number and thickness decreased significantly in long bones of the homozygous animals, whereas trabecular separation increased. In particular hypertrophic callus formation at fracture sites is reminiscent of type V OI $[1,2]$.

In summary, we were able to visualize an array of skeletal pathologies in Opt-mutant mice by the non-destructive approach of $\mu \mathrm{CT}$ imaging that qualitatively correlated with histological findings. In addition to commonly employed and thresholding-dependent 3D surface reconstructions the superior and additional information obtained by generating additional 2D MPRs was shown in an exemplary fashion and should be considered for future characterization of comparable animal models of skeletal disease.

Conflicts of interest The authors declare that there is no conflict of interest.

Open Access This article is distributed under the terms of the Creative Commons Attribution Noncommercial License which permits any noncommercial use, distribution, and reproduction in any medium, provided the original author(s) and source are credited.

\section{References}

1. Glorieux FH, Rauch F, Plotkin H, et al. Type V osteogenesis imperfecta: a new form of brittle bone disease. J Bone Miner Res. 2000;15:1650-8.

2. Sohaskey ML, Jiang Y, Zhao JJ, Mohr A, Roemer F, Harland RM. Osteopotentia regulates osteoblast maturation, bone formation, and skeletal integrity in mice. J Cell Biol. 2010;189:511-25

3. Studholme C, Hill DLG, Hawkes DJ. An overlap invariant entropy measure of 3D medical image alignment. Pattern Recognit. 1999;32:71-86.

4. Studholme C. Rview for windows. http://rview.colin-studholme.net. Accessed 17 November 2010.

5. Hildebrand T, Laib A, Müller R, Dequeker J, Ruegsegger P. Direct three-dimensional morphometric analysis of human cancellous bone: microstructural data from spine, femur, iliac crest, and calcaneus. J Bone Miner Res. 1999;14:1167-74.

6. Mohr A, Heiss C, Bergmann I, et al. Value of micro-CT as an investigative tool for osteochondritis dissecans. Acta Radiol. 2003;44:532-7.
7. Neues F, Epple M. X-ray microcomputer tomography for the study of biomineralized endo- and exoskeletons of animals. Chem Rev. 2008;108:4734-41.

8. Freeman TA, Patel P, Parvizi J, Antoci V, Shapiro IM. Micro-CT analysis with multiple thresholds allows detection of bone formation and resorption during ultrasound-treated fracture healing. J Orthop Res. 2009;27:673-9.

9. Langheinrich AC, Bohle RM, Greschus S, et al. Atherosclerotic lesions at micro CT: feasibility for analysis of coronary artery wall in autopsy specimens. Radiology. 2004;231:675-81.

10. Langheinrich AC, Leithauser B, Greschus S, et al. Acute rat lung injury: feasibility of assessment with micro-CT. Radiology. 2004;233:165-71.

11. Abe S, Watanabe H, Hirayama A, Shibuya E, Hashimoto M, Ide Y. Morphological study of the femur in osteopetrotic (op/op) mice using microcomputed tomography. Br J Radiol. 2000;73:1078-82.

12. Bergo MO, Gavino B, Ross J, et al. Zmpste24 deficiency in mice causes spontaneous bone fractures, muscle weakness, and a prelamin A processing defect. Proc Natl Acad Sci USA. 2002;99:13049-54.

13. Swain MV, Xue J. State of the art of Micro-CT applications in dental research. Int J Oral Sci. 2009;1:177-88.

14. Ford-Hutchinson AF, Cooper DM, Hallgrimsson B, Jirik FR. Imaging skeletal pathology in mutant mice by microcomputed tomography. J Rheumatol. 2003;30:2659-65.

15. Yamashita T, Sekiya I, Kawaguchi N, et al. Klotho-deficient mice are resistant to bone loss induced by unloading due to sciatic neurectomy. J Endocrinol. 2001;168:347-51.

16. Obenauer S, Alamo L, Herold T, Funke M, Kopka L, Grabbe E. Imaging skeletal anatomy of injured cervical spine specimens: comparison of single-slice vs multi-slice helical CT. Eur Radiol. 2002;12:2107-11.

17. Begemann PG, Kemper J, Gatzka C, Stork A, Nolte-Ernsting C, Adam G. Value of multiplanar reformations (MPR) in multidetector CT (MDCT) of acute vertebral fractures: do we still have to read the transverse images? J Comput Assist Tomogr. 2004;28:572-80.

18. Thornton MM. Multi-modality imaging of musculoskeletal disease in small animals. J Musculoskel Neuron Interact. 2004;4:364.

19. Schambach SJ, Bag S, Schilling L, Groden C, Brockmann MA. Application of micro-CT in small animal imaging. Methods. 2010;50:2-13.

20. Müller R. Hierarchical microimaging of bone structure and function. Nat Rev Rheumatol. 2009;5:373-81.

21. David V, Laroche N, Boudignon B, et al. Noninvasive in vivo monitoring of bone architecture alterations in hindlimb-unloaded female rats using novel three-dimensional microcomputed tomography. J Bone Miner Res. 2003;18:1622-31.

22. Guldberg RE, Lin AS, Coleman R, Robertson G, Duvall C. Microcomputed tomography imaging of skeletal development and growth. Birth Defects Res C Embryo Today. 2004;72:250-9.

23. Stauber M, Müller R. Micro-Computed Tomography: a method for the non-destructive evaluation of the three-dimensional structure of biological specimens. In: Westendorf JJ, editor. Methods in molecular biology. Totowa, NJ: Humana; 2008. p. 273-92. 